\title{
The Effect of the Narrow-Band Noise in the Range 4-8 Hz on the Alpha Waves in the EEG Signal
}

\author{
C. KASPRZAK*
}

AGH - University of Science and Technology, Faculty of Mechanical Engineering and Robotics

Department of Mechanics and Vibroacoustics, al. A. Mickiewicza 30, 30-059 Krakow, Poland

\begin{abstract}
The paper summarises the results of experiments carried out to investigate the effect of a narrow-band noise in the range $4-8 \mathrm{~Hz}$ and of the acoustic pressure SPL $=110 \mathrm{~dB}(\mathrm{HP})$ on the alpha waves in the EEG signal. The test group included 23 males aged $21-33$. It is found out that white noise in the infrasound range $4-8 \mathrm{~Hz}$ leads to changes of amplitudes of the spectral power density of alpha waves. The tests reveal a statistically significant reduction of the alpha wave amplitude during the infrasound exposure and an amplitude increase when the exposure is over.
\end{abstract}

DOI: 10.12693/APhysPolA.123.980

PACS: 87.50.Y-, 43.28.Dm, 87.19.lr

\section{Introduction}

From the standpoint of physics, the critical frequency of infrasounds $(20 \mathrm{~Hz})$ suggests that they should be well beyond the audibility range and hence will not be heard. This view appears to be rather imprecise, however, because at sufficiently high levels of acoustic pressure, infrasounds are received by the ear and by the vestibular system. Generally, the auditory threshold of infrasound perception is determined through experiments on otologically sound young people and is obtained as the median of the auditory threshold levels registered for individual test participants.

It follows, therefore, that under specific conditions the perception of infrasounds in the frequency range $4-8 \mathrm{~Hz}$ is quite possible [1]. Infrasounds may be received not only through the ear. Human body has a number of receptors enabling the perception of acoustic waves in the frequency range below $20 \mathrm{~Hz}$. On the skin surface there are numerous vibration and touch detectors operating in various ranges of reference frequency.

In his paper published in Noise and Vibration Bulletin, Hartmut Ising summarised the results of his experiment that he had carried out in 1980 [2]. The participants were exposed to infrasounds in the frequency ranges: $3-6 \mathrm{~Hz}$, $6-12 \mathrm{~Hz}, 12-24 \mathrm{~Hz}$ and with intensity of $110 \mathrm{~dB}$ or to a combination of sounds in the frequency range $6-12 \mathrm{~Hz}$. Tests revealed that psychological tension in participants tends to increase with frequency and the concentration deteriorates. No effect on the response time, breathing rate, or pulse rate was established.

In 1992 Arabadzhi published the results of his experiment aimed to establish the influence of infrasounds produced by swelling sea or ocean waves on biological rhythms of the human brain [3]. He established the connection between the frequency of the alpha waves in the

*e-mail: cekasp@agh.edu.pl human brain and the frequency of the applied infrasounds and on that basis formulated the recommendations for retaining the waking state.

The effects of infrasound noise on the human body have been recognised to a certain extent. Experimental data reveal variations in the circulatory systems, variations of bioelectric activity of the human brain during the infrasound exposure, and changes in the level of activation [4-6].

One purpose of the experiment was to determine the effect of infrasound waves on variations in the alpha waves. Tests were carried out on a group of 32 participants. The experiment showed that infrasounds of frequency $f=7 \mathrm{~Hz}$ and acoustic pressure level $=120 \mathrm{~dB}(\mathrm{HP})$ cause a statistically significant reduction of the alpha rhythm power [7].

The alpha waves were first registered and named by Berger in 1929. They involve rhythmic activity of the cerebral cortex in the frequency range 8-12 Hz. The occurrence of the alpha rhythm is attributed to the wakeful relaxation with the eyes closed. Alpha waves, best manifested in the occipital region in humans remaining with their eyes closed, have a variable amplitude, about $50 \mathrm{mV}$ on the average. They evidence the synchronisation of activities of numerous dendrite units and appear during wakeful relaxation. The frequency of alpha waves changes but slightly, starting from early childhood right through to the age of maturity $[8,9]$.

\section{Methodology of research}

The test group included 23 males aged 21-33. The examined individuals were volunteers who declared that they had not any medical conditions and were not under the influence of medicines. They had been also informed that before the experiment, they were not allowed to drink any stimulating nor intoxicating drinks. In the research, infrasounds were used with frequency from 4 up to $8 \mathrm{~Hz}$ and the sound pressure level SPL $=110 \mathrm{~dB}(\mathrm{HP})$. The total duration of the experiment was $35 \mathrm{~min}$. The 
exposure of the stimulus was 20 min. The acoustic signal, recorded in a wave format, was played from a computer.

After conducting preparation activities, a proper experiment took place; that is 35 min of constant acquisition of EEG human bioelectric signals. The initial 5 min was recorded without the infrasound exposure, 20 min with the signal exposure, and 10 min without the exposure [10]. The study was conducted with the approval of the Local Commission of Bioethics in Łódż.

The experimental set up comprised:

- a stimulus-generating circuit,

- a Hungarian-type pressure chamber,

- the circuit for recording and analysis of EEG signals.

The signal from the WAV-type file was amplified by the electric amplifier ELMUZ 2158M and fed to 6 amplifiers GDN 30/80/2 installed in the ceiling in the infrasound chamber. The acoustic signal applied in the experiment was the narrow-band white noise in the frequency range restricted to $4-8 \mathrm{~Hz}$ and $\mathrm{SPL}=110 \mathrm{~dB}(\mathrm{HP})$ (Fig. 1).



Fig. 1. Acoustic spectrum of the applied infrasound noise.

The EEG test consisted in registration (with a help of electrodes placed on the skin of a head) of functional currents of a human brain characteristic feature of which is small tension (from several to several hundred microvolts). The frequency of these currents ranges from $0.5 \mathrm{~Hz}$ to $50 \mathrm{~Hz}$. The registration of EEG signal was conducted with a help of 25-channel box of SAM 25 type of MICROMED Company. EEG cap was fitted in accordance with a standard $10 / 20$ system, where electrodes are placed along the sagittal line of the head: 5 on the left side (Fp1, F3, C3, P3, O1), 5 on the right side (Fp2, F4, C4, P4, O2), and a reference electrode $(\mathrm{OP}, \mathrm{Pz})$. The EEG signal is registered with the sampling frequency $256 \mathrm{~Hz}$, using the application 'System98BrainQuick'. For each participant we obtained a set of 10 plots of the power spectral density function. Each plot (five for each hemisphere) represents the record registered by one of the electrodes. Variations of the alpha rhythm were analysed by performing the variance analysis ANOVA between the subsequent stages of the test procedure. Fluctuations of the average amplitude of the alpha wave between the subsequent stages analysed using the $t$-Student test.

\section{Result analysis}

The qualitative analysis of the power spectral density plots was carried out to establish whether infrasound exposure would cause the alpha rhythm to change. Changes of the EEG signal during the infrasound exposure were registered for 15 participants ( $75 \%$ of the test group). In the case of 14 men ( $70 \%$ of the test group), the applied acoustic stimuli gave rise to a significant reduction of the alpha rhythm and in a majority of them ( 8 people, $40 \%$ of the test group) the amplitude of alpha waves would return to the level registered in the first stage of the procedure, after the exposure to the acoustic stimuli. In three persons ( $15 \%$ of the test group), the amplitude of the alpha wave was further reduced until the series of the testing was over. Further, the dominating frequency of the alpha rhythm was found to be changed in 8 persons (40\% of the test group).

The qualitative analysis was followed by a quantitative assessment of the effects of the infrasound exposure on the alpha rhythm. The alpha waves were in the frequency range $8-13 \mathrm{~Hz}$.

Alpha waves are registered mainly in the parietooccipital region and have a variable amplitude, in the range of $50 \mu \mathrm{V}$. They occur as a result of synchronised activities of numerous dendritic units and appear in the waking and relaxed state. They are typically registered when the eyes are closed and tend to disappear during the intellectual effort, for instance when solving mathematical problems or after opening eyes, when they see the light. As regards alpha waves, the hypothesis was to be verified that would assume good agreement between their averaged amplitudes and the normal distribution. This hypothesis was found to hold good, hence the distribution of the variables should be normal $(p>0.05)$. The single-factor variance analysis ANOVA was performed. It was found that during the second stage of the experiment (infrasound exposure) the amplitude of the alpha waves would significantly decrease (Fig. 2).

In relation to levels registered at stage I, the average value decreases by about $13 \%(p<0.05)$. Further, it appears that after the infrasound exposure is ceased, the alpha rhythm would return to the levels registered before the stage II of the experiment $(p<0.05)$. It is reasonable to conclude, therefore, that exposure to infrasounds in the form of white noise in the frequency range $4-8 \mathrm{~Hz}$ would strongly influence the alpha rhythm in test participants (Table I).

The analytical procedure involved the study of fluctuations of the mean amplitude of the power spectral density of alpha waves between the three subsequent stages of the experiment. Since the underlying distribution of averaged values of the alpha wave amplitudes turned out to be normal, the independent variables were treated using the $t$-Student test (Table II). 


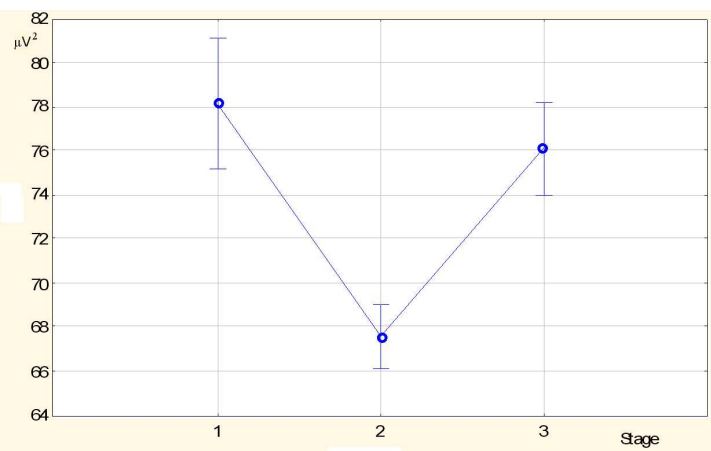

Fig. 2. Variations of the alpha rhythm power $\left(\mu \mathrm{V}^{2}\right)$ for EEG in particular stages of the experiment: Stage 1 prior to infrasound exposure; Stage 2 - infrasound exposure; Stage 3 - after the infrasound exposure. Designations used: the mean and the mean \pm standard error, mean $\pm 1.96 \times$ standard error.

TABLE I

Significance of differences of alpha rhythm power between particular stages of the experiment. ANOVA test statistics: $S S$ - sum of squares, $D F$ - degrees of freedom, $M S$-mean square, $F-F$-ratio, $p-p$-value $\left({ }^{*}\right.$ statistical significance $p<0.05$ ).

\begin{tabular}{l|r|r|r|r|c}
\hline \hline & \multicolumn{1}{|c|}{$S S$} & \multicolumn{1}{c|}{ DF } & \multicolumn{1}{c|}{$M S$} & $F$ & $p$ \\
\hline $\begin{array}{c}\text { Absolute } \\
\text { term }\end{array}$ & 840428.6 & 1 & 840428.6 & 12355.25 & $0.00^{*}$ \\
\hline Stage & 4414.7 & 2 & 2207.4 & 32.45 & $0.00^{*}$ \\
\hline Error & 14080.5 & 207 & 68.0 & &
\end{tabular}

Table II summarises the differences in significance levels between particular stages of the experiment for EEG. Stage 1-2, Stage 1-3, Stage 2-3 denote comparable time intervals, $\mathrm{p}$ - significance level test.

Figure 3 shows the change of alpha rhythm power $\left(\mathrm{pV}^{2}\right)$ between Stage 1 (no exposure) and Stage 2 (with infrasound exposure). A significant decrease of alpha rhythm power was observed. At the end of infrasound exposure (Stage 3), a statistically significant increase of alpha rhythm power was found compared to Stage 2 (with infrasound exposure) (Fig. 4 and Table II).

There were no significant changes between Stages 1 and 3 (Fig. 5 and Table II).

The decrease of the alpha wave amplitude was registered during the infrasound exposure whilst this param-

\section{TABLE II}

Significance of differences of alpha rhythm power between particular stages of the experiment. $t$-Student test statistics: $t-t$-statistic, $d f-$ degrees of freedom, $p-p$-value ( ${ }^{*}$ statistical significance $\left.p<0.05\right)$.

\begin{tabular}{l|r|r|r|r|c}
\hline \hline Stage & mean-1 & mean-2 & \multicolumn{1}{c|}{$t$} & \multicolumn{1}{c|}{ df } & $p$ \\
\hline $1-2$ & 78.13 & 67.75 & 6.21 & 148 & $0.00^{*}$ \\
\hline $2-3$ & 67.75 & 75.62 & -6.22 & 178 & $0.00^{*}$ \\
\hline $1-3$ & 78.13 & 75.62 & 1.20 & 88 & 0.23
\end{tabular}

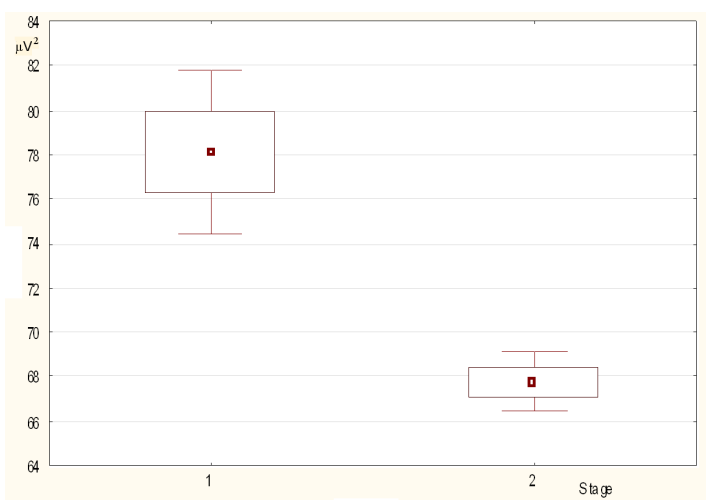

Fig. 3. Differences of alpha rhythm power $\left(\mu \mathrm{V}^{2}\right)$ between particular stages of the experiment: Stage $1-$ prior to infrasound exposure; Stage 2 - infrasound exposure. Designations used: the mean and the mean \pm standard error, mean $\pm 1.96 \times$ standard.

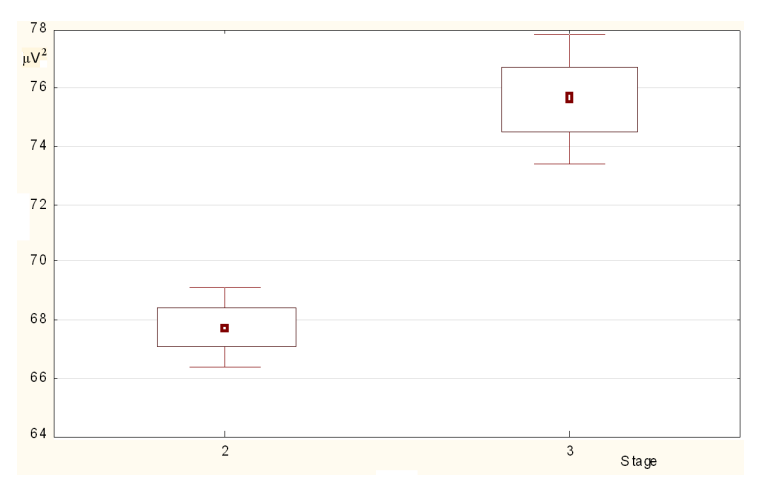

Fig. 4. Differences of alpha rhythm power $\left(\mu \mathrm{V}^{2}\right)$ between particular stages of the experiment: Stage $2-$ infrasound exposure; Stage 3 - after the infrasound exposure. Designations used: the mean and the mean \pm standard error, mean $\pm 1.96 \times$ standard error.

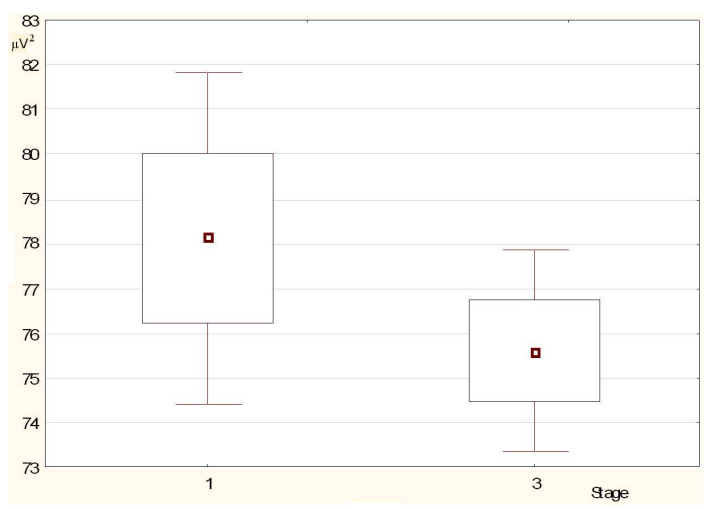

Fig. 5. Differences of alpha rhythm power $\left(\mu \mathrm{V}^{2}\right)$ between particular stages of the experiment: Stage 1 prior to infrasound exposure; Stage 3 - after the infrasound exposure. Designations used: the mean and the mean \pm standard error, mean $\pm 1.96 \times$ standard error. 
eter tends to increase once the infrasound exposure is over. The alpha blockage is a well-known effect caused by desynchronised bioelectrical activity of the brain under the sensory stimulation. This phenomenon is registered even though the stimulus applied is an acoustic stimulus not perceived directly by the aural system. Reduction of the alpha rhythm during the infrasound exposure is found on the statistical significance level $p<0.05$.

\section{Conclusions}

The registered EEG patterns were subject to a qualitative analysis involving the assessment of changes of the power spectral density of investigated signals under the exposure to acoustic stimuli. Besides, the quantitative analysis was performed to determine the influence of the infrasound exposure on the amplitude of the power spectral density function of the major biological rhythms in the human brain and the level of the test participants' excitability.

In the case of $70 \%$ of the test group, these changes were revealed as the decrease of the alpha wave amplitude (for 8 people, the amplitude of the alpha wave went up again after the infrasound exposure; for 3 people, further decrease in the Stage 3 of the experiment; for 1 person, the alpha wave amplitude after the infrasound exposure remained on the same level as during the exposure). Furthermore, in three men subject to infrasound exposure, the frequency of the alpha wave increased ( $5 \%$ of the test group).
Considerable decrease of the alpha wave amplitude during the infrasound exposure and increase in the value of this parameter once the exposure is over was observed.

\section{Acknowledgement}

This study is a part of the N N501 247740 research project supported by the National Science Centre.

\section{References}

[1] N.S. Yeowart, W. Bryan, M.E. Tempest, J. Sound Vibrat. 6, 335 (1967).

[2] H. Ising, Noise Vib. Bull. 8, 168 (1980).

[3] V.I. Arabadzhi, Biofizika 37(1), 150 (1992).

[4] C. Kasprzak, Acta Phys. Pol. A 118, 87 (2010).

[5] C. Kasprzak, Acta BioOptica Inform. Med. 15, 390 (2009).

[6] Z. Damijan, C. Kasprzak, R. Panuszka, J. Acoust. Soc. Am. 115, 2388 (2004).

[7] C. Kasprzak, Acta Phys. Pol. A 121, A-61 (2012).

[8] F. Boiten, J. Sergeant, R. Geuze, Electroencephalogr. Clin. Neurophysiol. 82, 302 (1992).

[9] C. Grillon, M.S. Buchsbaum, Electroencephalogr. Clin. Neurophysiol. 63, 42 (1986).

[10] C. Kasprzak, Acta Phys. Pol. A 119, 986 (2011). 